\title{
Mental health-related stigma and pathways to care for people at risk of psychotic disorders or experiencing first-episode psychosis: a systematic review
}

\author{
P. C. Gronholm ${ }^{1 *}$, G. Thornicroft ${ }^{1,2,3}$, K. R. Laurens ${ }^{4,5,6,7}$ and S. Evans-Lacko ${ }^{1,2,8}$ \\ ${ }^{1}$ Health Service and Population Research Department, Institute of Psychiatry, Psychology \& Neuroscience, King's College London, London, UK \\ ${ }^{2}$ Centre for Global Mental Health, Institute of Psychiatry, Psychology \& Neuroscience, King's College London, London, UK \\ ${ }^{3}$ Centre for Implementation Science, Institute of Psychiatry, Psychology \& Neuroscience, King's College London, London, UK \\ ${ }^{4}$ Department of Forensic and Neurodevelopmental Science, Institute of Psychiatry, Psychology \& Neuroscience, King's College London, London, UK \\ ${ }^{5}$ School of Psychiatry, University of New South Wales, Sydney, New South Wales, Australia \\ ${ }^{6}$ Schizophrenia Research Institute, Sydney, New South Wales, Australia \\ ${ }^{7}$ Neuroscience Research Australia, Sydney, New South Wales, Australia \\ ${ }^{8}$ Personal Social Services Research Unit, London School of Economics and Political Science, London, UK
}

Background. Stigma associated with mental illness can delay or prevent help-seeking and service contact. Stigmarelated influences on pathways to care in the early stages of psychotic disorders have not been systematically examined.

Method. This review systematically assessed findings from qualitative, quantitative and mixed-methods research studies on the relationship between stigma and pathways to care (i.e. processes associated with help-seeking and health service contact) among people experiencing first-episode psychosis or at clinically defined increased risk of developing psychotic disorder. Forty studies were identified through searches of electronic databases (CINAHL, EMBASE, Medline, PsycINFO, Sociological Abstracts) from 1996 to 2016, supplemented by reference searches and expert consultations. Data synthesis involved thematic analysis of qualitative findings, narrative synthesis of quantitative findings, and a meta-synthesis combining these results.

Results. The meta-synthesis identified six themes in relation to stigma on pathways to care among the target population: 'sense of difference', 'characterizing difference negatively', 'negative reactions (anticipated and experienced)', 'strategies', 'lack of knowledge and understanding', and 'service-related factors'. This synthesis constitutes a comprehensive overview of the current evidence regarding stigma and pathways to care at early stages of psychotic disorders, and illustrates the complex manner in which stigma-related processes can influence help-seeking and service contact among first-episode psychosis and at-risk groups.

Conclusions. Our findings can serve as a foundation for future research in the area, and inform early intervention efforts and approaches to mitigate stigma-related concerns that currently influence recognition of early difficulties and contribute to delayed help-seeking and access to care.

Received 26 September 2016; Revised 18 January 2017; Accepted 24 January 2017; First published online 15 February 2017

Key words: Discrimination, early intervention, FEP, healthcare-seeking behaviour, healthcare utilization, help-seeking behaviour, labelling, literature review, stigma, treatment barriers.

\section{Introduction}

Psychotic disorders can have a severe or long-term impact (Tandon et al. 2009). Although timely access to treatment is associated with improved outcomes (Birchwood \& Macmillan, 1993; McGorry et al. 1996),

* Address for correspondence: Dr P. C. Gronholm, Health Service and Population Research Department (Box P029), Institute of Psychiatry, Psychology \& Neuroscience, King's College London, De Crespigny Park, London SE5 8AF, UK.

(Email: petra.gronholm@kcl.ac.uk) there is often considerable delay between initial onset of psychotic symptoms and treatment initiation (Ho \& Andreasen, 2001). Early intervention services aim to improve clinical and social outcomes through reducing the duration of untreated psychosis (DUP) (Birchwood et al. 1997; McGorry et al. 2000). It is also possible to intervene preventatively, prior to the onset of frank psychotic symptoms, through strategies targeting people at clinically defined increased risk of developing psychotic disorder (Kohler et al. 2014). These early intervention efforts - whether targeting

This is an Open Access article, distributed under the terms of the Creative Commons Attribution licence (http://creativecommons.org/licenses/by/4.0/), which permits unrestricted re-use, distribution, and reproduction in any medium, provided the original work is properly cited. 
first-episode psychosis (FEP) or clinical high-risk states build on the premise of reduced service access delays. Emerging evidence supports the utility of these strategies (Preti \& Cella, 2010; Stafford et al. 2013; Castle \& Singh, 2015).

To improve timely access to support, greater understanding of how to facilitate help-seeking and identification of potential barriers in the processes underpinning service contact is needed. Stigma associated with mental illness is one such potential influence. Stigma has been defined as the co-occurrence of processes reflecting labelling, stereotyping, separating, emotional reactions, and status loss and discrimination, within a power context favouring the stigmatizer (Link et al. 2004). These processes can operate in a number of settings, and are evident through various direct and indirect social interactions. Different types of stigma have been formulated to describe these various manifestations. For example, it is proposed that stigma can be defined in terms of how its influence is experienced, or using an action-orientated perspective of who (or what) gives or receives the stigma (Pescosolido \& Martin, 2015). Experiential types of stigma include perceived stigma (considering beliefs 'most people' are thought to hold), endorsed stigma (expressed agreement with stereotypes/prejudice/discrimination), anticipated stigma (expected experiences of prejudice/discrimination), received stigma (overt experiences of rejection or devaluation), or enacted stigma (discriminatory behaviours). Action-orientated ways of considering stigma recognize, for example, public stigma (stereotypes, prejudice and discrimination endorsed by the general population), structural stigma (prejudice and discrimination through laws, policies, and constitutional practices), courtesy stigma (stereotypes, prejudice, and discrimination acquired through an association with a stigmatized group/person), provider-based stigma (prejudice and discrimination by occupational groups designated to provide assistance to stigmatized groups), and internalized stigma (when people who belong to a stigmatized group legitimize publicly held stereotypes and prejudice, and internalize these by applying them to themselves). People's preference to avoid stigma, instigated by receiving a mental illness diagnosis or through association with mental health services, may delay or prevent helpseeking and service contact. These influences have been explored in narrative reviews (Corrigan, 2004; Schomerus \& Angermeyer, 2008; Thornicroft, 2008) and one systematic review (Clement et al. 2015) that considered various populations and mental health conditions. All reported evidence that stigma can constitute a barrier to help-seeking and service use.

Stigma-related influences on pathways to care might be pertinent in relation to help-seeking and accessing care for FEP or during at-risk states of psychotic disorders, as psychosis is a highly stigmatized condition both in terms of its diagnosis and associated symptoms (Rose et al. 2011; Yang et al. 2013; Lasalvia et al. 2014). To our knowledge, there have been no systematic efforts to examine stigma in relation to help-seeking and service contact among these two specific populations. Improved understanding of these influences could inform efforts to mitigate stigma-related barriers to help-seeking and service use in early stages of psychotic disorders.

This review aimed to systematically assess findings from qualitative, quantitative and mixed-methods research (MMR) studies examining the relationship between stigma and pathways to care (term denoting processes associated with help-seeking and health service contact). The primary objective was to examine this relationship among people experiencing FEP or at risk of a psychotic disorder, and among significant other individuals supporting their pathways to care (e.g. through help-seeking assistance and initiating service contact). As secondary objectives, the review explored possible mechanisms through which stigma was reported to influence pathways to care, and how well researched stigma was in relation to these pathways.

\section{Method}

As per recommendations for research questions where both qualitative and quantitative evidence is available (Oliver et al. 2005; EPPI Centre, 2010), this systematic mixed-studies review separately considered qualitative and quantitative evidence before merging these data. The review protocol was developed a priori and registered at PROSPERO Centre for Reviews and Dissemination (ID CRD42014009206), and followed the Preferred Reporting Items for Systematic Reviews and Meta-Analyses (PRISMA) statement (Moher et al. 2009); see online Supplement 1.

\section{Search strategy and selection of studies}

Five electronic databases (CINAHL, EMBASE, Medline, PsycINFO, Sociological Abstracts) were searched in July 2016, for papers published between 1996 and 2016. Subject headings and keywords were related to the following terms: at-risk status/FEP AND stigma AND help-seeking/service use (see online Supplement 2 for the full search strategies). The searches were limited to 'human' publications in English, and studies considering people aged up to and including 40 years. Backward and forward reference searches were carried out for all relevant papers identified through the database searches, and authors 
of these papers and other content experts were contacted for recommendations regarding additional publications. Initial result screening considered studies' titles, abstracts and keywords. To establish consistency, $15 \%$ of these results were independently screened by the main author and a second rater, and discrepancies resolved via discussion. Full-text reports were obtained for all potentially relevant studies. Inclusion criteria (see Table 1) were data-based, peer-reviewed articles concerning stigma in relation to pathways to care among people at risk of developing a psychotic disorder or experiencing FEP, using qualitative, quantitative, or MMR approaches.

Studies met the stigma inclusion criteria if the findings were explicitly described as 'stigma', and also if a study reported on processes (e.g. thoughts, beliefs, inter- or intrapersonal dynamics, experienced behaviours) that were not explicitly defined using the term 'stigma' but were reflective of the processes underpinning stigma: labelling, stereotyping, separating, emotional reactions, and status loss and discrimination, within a power context favouring the stigmatizer (Link et al. 2004).

\section{Data extraction, analysis and synthesis}

Data were extracted on study design, population characteristics, and summary descriptions on stigma and pathways to care. For qualitative studies, relevant data were participant quotes and authors' interpretations and summaries regarding stigma in relation to pathways to care, as reported in study results sections. For quantitative studies, details were extracted regarding how the study operationalized stigma/discrimination and pathways to care, and data on the connection between these (e.g. association between stigma and help-seeking variables, prevalence of stigma-related help-seeking barriers). For MMR studies, summary descriptions were extracted for qualitative and quantitative findings, alongside details on how these were related.

Studies' methodological quality was assessed using the Mixed Methods Appraisal Tool (MMAT; Pluye et al. 2011). The MMAT assesses two generic core quality criteria, and methodology-specific aspects that include four quality dimensions for qualitative and quantitative designs and three dimensions for MMR designs. Articles were assigned 1 point for each dimension where criteria was met, and half a point for partially met criteria. These points were summed for an overall score, ranging from $0 \%$ (no criteria met) to $100 \%$ (all criteria met).

Data synthesis was conducted in three stages. First, thematic analysis (Braun \& Clarke, 2006) was undertaken to synthesize the findings of articles reporting qualitative data. Data were extracted verbatim from the articles, and transferred into qualitative analysis software (NVivo 10; QSR International). An initial coding frame was developed based on inductive open coding. Data were iteratively indexed and sorted within this, and themes continuously related and restructured, until a thematic framework which accurately and comprehensively reflected the data emerged. This process was validated through consensus meetings between authors (P.C.G., S.E-L.). Themes within the framework were connected if at least one article reported a relationship between them.

The second stage involved a narrative synthesis (Popay et al. 2006) of the findings of the articles reporting on quantitative data. Synthesis involved assessing quantitative findings regarding stigma and pathways to care across studies, and summarizing these within a textual narrative.

The third stage involved a meta-synthesis to bring together the multi-level insights from the qualitative and quantitative syntheses (Centre for Reviews and Dissemination, 2009). This approach juxtaposes, rather than pools, findings from the separate syntheses into an overall picture reflecting commonalities, also highlighting differences where relevant (Pope et al. 2007). An overarching conceptual meta-synthesis framework was produced by examining the quantitative synthesis results in relation to the thematic framework derived from the qualitative synthesis. Subthemes from the quantitative synthesis were integrated into the thematic model, indicated using underlining. This provided an overview of the overall results while delineating the derivation of synthesis themes, enabling comparing and contrasting these findings.

\section{Additional analyses}

\section{Sensitivity analysis}

To examine how the studies' methodological quality might have influenced the review findings, articles meeting $\leqslant 50 \%$ of MMAT criteria were excluded from the meta-synthesis model to assess whether this changed the results.

\section{Subgroup analyses}

To further examine the association between stigma and pathways to care, subgroup comparisons were planned among: (1) people at risk of developing psychotic disorders, $v$. those experiencing FEP; (2) people affected by at-risk stages of psychotic disorders or $\mathrm{FEP}, v$. significant other individuals; and (3) significant other individuals of people at risk of developing psychotic disorders, $v$. significant other individuals of 
Table 1. Inclusion and exclusion criteria for articles included in the systematic review

\author{
Population \\ First-episode psychosis or at-risk \\ stages of psychosis \\ Include
}

Exclude

\section{Domains studied \\ Stigma \\ Include}

Exclude

Pathway to care

Include

Exclude

Person reporting on pathway

to care

Include

Exclude

\section{Study type}

Articles reporting on data-based, peer-

reviewed qualitative, quantitative or mixed methods research studies Include

Exclude

\section{Other criteria}

Year of publication

Include

Exclude

Language

Include

Exclude

Type of research

Include

Exclude
People experiencing first-episode psychosis; or experiencing attenuated psychotic-like symptoms below the threshold of frank psychotic symptoms but indicative of an increased risk of developing schizophrenia and other psychotic disorders; or people reporting an early illness presentation characterized by the presence of subclinical psychotic-like experiences (PLEs). People experiencing these difficulties aged up to and including 40 years of age

People in at-risk stages based on a genetic or familial high risk for psychosis or schizophrenia; or people experiencing chronic psychosis or disorders other than (early) psychotic disorders/schizophrenia. People over the age of 40 years

Findings described as, or reflective of, any kind of stigma related to mental health; including experienced stigmas (perceived, endorsed, anticipated, received, or enacted) or action-orientated stigmas (public stigma, structural stigma, courtesy, and internalized-stigma)

Stigmas relating to attributes other than mental health, for example other health conditions such as HIV, gender, sexuality, nationality, ethnic origin

Processes of and features within help-seeking; service contact/use; or periods of untreated illness prior to/during help-seeking, or prior to service contact Articles that consider pathways to care in association with mental health problems which are not characterized as early of symptoms and signs of psychosis (including chronic psychosis or schizophrenia); substance abuse; dementia; intellectual disabilities

Articles where pathways to care are reported by either the person affected by first-episode/early psychosis or at-risk stages of psychosis, or a significant other person assisting the individual or initiating service contact on behalf of the affected person

Articles where pathways to care are reported by other people not listed above (e.g. formal service providers, people acting within a professional service provider capacity)

Data-based, peer-reviewed articles that use qualitative, quantitative or mixed methods research approaches to assess stigma in relation to pathways to care within the population of interest

Non-data based or non-peer-reviewed articles; for example, reviews, research protocols, editorials, comments, letters and dissertations

Articles published between January 1996 and July 2016

Articles published prior to 1996

Articles published in English

Articles not published in English

Articles identified using the 'human' search filter

Animal studies, and any other type of research not included in the 'human' search filter 
those experiencing FEP. For these comparisons, the relative proportions of articles reporting on stigmarelated themes were compared across the subgroups. We considered findings of articles where stigma-related thoughts and experiences were discussed as related pathways to care, or where a statistically significant association was reported between measures reflecting stigma and pathways to care.

\section{Results}

The search produced 8544 non-duplicated results, of which 8330 were excluded following initial screening. Full-text articles were accessed for the remaining 214 references; 171 articles were excluded following assessment against full eligibility criteria, and three articles were excluded later upon further inspection of methodological details. Forty articles met review inclusion criteria. Fig. 1 depicts this process; full details on the included articles are provided in online Supplement 3 , and online Supplement 4 lists the excluded full-text articles.

Most included articles described qualitative studies $(77.5 \%, 31 / 40)$; fewer quantitative $(17.5 \%, 7 / 40)$ and MMR $(5.0 \%, 2 / 40)$ papers were included. Three-quarters (77.5\%, 31/40) considered FEP populations. Around half of the studies $(55.0 \%, 22 / 40)$ considered the perspective of the affected person (i.e. person at risk of psychosis or experiencing FEP), just under a third the perspective of significant others $(30.0 \%, 12 / 40)$, and six $(15.0 \%)$ considered a joint perspective of both. When considering these core study characteristics jointly - population and person describing pathway to care - most often articles considered the perspectives of people experiencing FEP (37.5\%, 15/40), followed by the perspectives of significant others of people affected by FEP (30.0\%, $12 / 40)$, and the perspectives of people in at-risk groups $(22.5 \%, 9 / 40)$.

The methodological quality of the included qualitative studies was overall good; MMAT ratings ranged from $37.5 \%$ to $100.0 \%$, but nearly all met $>50.0 \%$ of the criteria $(87.1 \%, 27 / 31)$. The most common limitations were not providing information on people who declined participation, and not discussing how results related to the research context or researcher's influence. The quality of the quantitative studies was mixed; MMAT ratings ranged from $37.5 \%$ to $100.0 \%$, with $50.0 \%$ the modal rating $(42.9 \%, 3 / 7)$. In quantitative studies, quality was compromised by limited reporting on response rates and sample representativeness. The MMR studies were of relatively poorer methodological quality, with MMAT ratings of $0.0 \%$ and $37.5 \%$ (rating reflects the weakest of qualitative, quantitative and MMR-specific scores). Both studies were compromised, for example, by limited critical consideration of integrating qualitative and quantitative components.

\section{Qualitative synthesis}

Thirty-three articles reported qualitative findings $(n=31$ qualitative studies, $n=2 \mathrm{MMR}$ ); considering data from 541 people. Most articles considered FEP groups (81.8\%, $27 / 33)$, four $(12.1 \%)$ considered clinically defined at-risk groups, and two $(6.1 \%)$ considered individuals at risk by virtue of experiencing auditory hallucinations that were distressing and/or precipitants of health service contact. Just over half $(54.5 \%, 18 / 33)$ considered the perspective of the affected person.

Six themes and 23 subthemes describing stigma and pathways to care among people experiencing FEP or at risk of psychosis were identified. These themes were: 'sense of difference', 'characterizing differences negatively', 'negative reactions (anticipated and experienced)', 'strategies', 'lack of knowledge and understanding', 'service-related factors'. A visual overview of these themes and their interconnections is provided in the conceptual model illustrated in Fig. 2, which also reports their frequencies (number of studies).

The first theme described experiences of a 'sense of difference' in relation to people at early stages of psychotic disorders, and the factors this difference was attributed to. This was captured in three subthemes, reflecting (i) a broad distinction of difference based on the impression that something was wrong, or not normal, to difference described in terms of both (ii) a general conceptualization of mental illness and (iii) more specific thoughts on particular diagnoses and descriptions of symptoms. The second theme of 'characterizing differences negatively' described how people considered different were often labelled with negative meanings and qualities, reflecting stereotyped beliefs. These characterizations were captured in three subthemes, reflecting (i) stigmatizing labels like mad, crazy or mental, and thoughts around a person being (ii) dangerous, violent, or unpredictable, or (iii) stupid, incapable or lazy. The third theme on 'negative reactions' captured anticipated and experienced responses - both from others and oneself - in relation to the sense of difference and the associated labelling. These were grouped into six subthemes, reflecting others' (i) negative and judgemental reactions, (ii) social distancing, (iii) sense of stigma, shame and embarrassment; and also personal feelings of (iv) shame and embarrassment, (v) guilt, and (vi) fear that experiences would worry or upset others. The fourth theme on 'strategies' captured people's attempts to avoid these reactions. Five subthemes described these efforts, covering (i) non-disclosure, (ii) concealment efforts, (iii) denying, ignoring, not accepting or admitting the situation, 


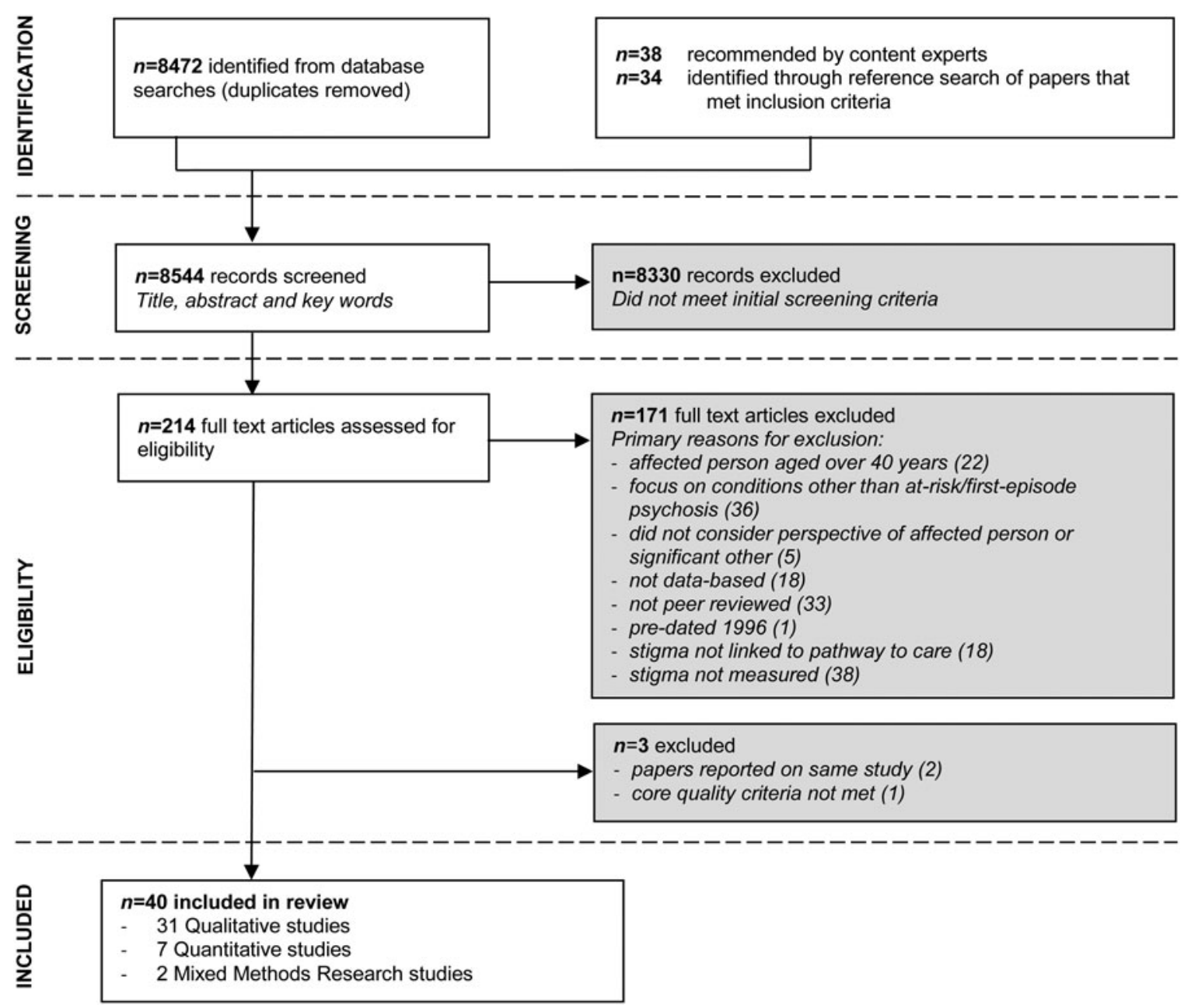

Fig. 1. Preferred Reporting Items for Systematic Reviews and Meta-Analyses (PRISMA) flow diagram summarizing the article selection process used in the systematic review.

(iv) normalizing and rationalizing experiences, and (v) social withdrawal. In terms of impact on pathways to care, the stigma-related experiences and beliefs described in these four themes were reported to contribute to, for example, reluctance to recognize symptoms, delayed help-seeking due to withholding difficulties from informal sources of support, and by influencing how people discussed, approached or engaged with services. The fifth theme, 'lack of knowledge and understanding', described how stigmarelated factors had contributed towards a limited awareness and understanding of mental illness, which consequently also compromised appropriate help-seeking. The sixth theme, 'service-related factors', described instances where deterring experiences of stigma were directly attributed to the service context, captured in the subthemes (i) feeling labelled, judged and treated differently by service providers; (ii) prejudiced attitudes towards and (fear of) mental health services; and (iii) belief that services break families apart.
However, this theme also described facilitative experiences where aspects of services had contributed to diminished stigma, captured in the subthemes (iv) normalizing, destigmatizing peer-environment, and (v) normalizing impact of treatment.

Online Supplement 5 illustrates these themes and subthemes further through participant quotes, as reported in the articles considered for this synthesis.

\section{Quantitative synthesis}

Nine articles reported on quantitative findings $(n=7$ quantitative studies, $n=2 \mathrm{MMR}$ ); reflecting data from 692 people. Five $(55.6 \%)$ considered FEP populations, three $(33.3 \%)$ groups defined through clinical high-risk criteria, and one $(11.1 \%)$ young people experiencing auditory hallucinations. Most articles $(55.6 \%, 5 / 9)$ considered affected persons' perspectives.

Six articles examined multivariate and bivariate associations between pathways to care and various 


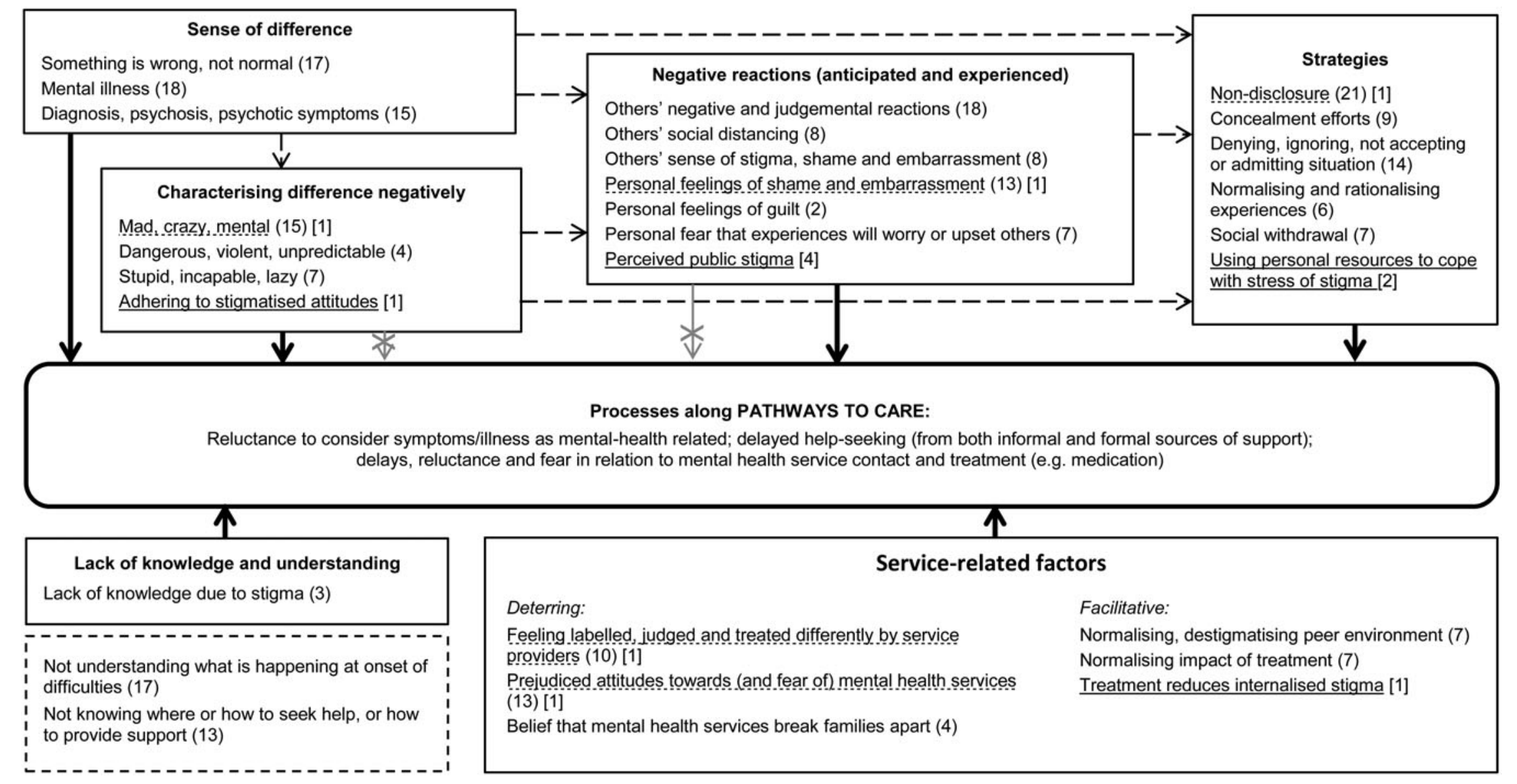

Fig. 2. Conceptual model based on meta-synthesis of qualitative $(n=33)$ and quantitative $(n=9)$ results. Boxes with solid outlines represent themes and subthemes. The box with the dashed outline lists processes which were not explicitly linked to stigma, but were related to a stigma-linked theme. Numbers in parentheses indicate the number of studies in the qualitative synthesis, and square brackets indicate the number of studies in the quantitative synthesis, that reported a subtheme. Underlined subthemes were reported in quantitative studies only, and dotted underlined subthemes were reported in both qualitative and quantitative studies. Dashed arrows indicate connections between the themes, heavy arrows indicate a link between a theme and pathways to care, and crossed-out arrows indicate a non-significant association with a pathway to care-related outcome. 
conceptualizations of stigma: perceived stigma (Compton \& Esterberg, 2005; Compton et al. 2009; Rüsch et al. 2013; Xu et al. 2016), stigma stress (Rüsch et al. 2013; Xu et al. 2016), stigmatized attitudes (Czuchta \& McCay, 2001), and internalized stigma (Morrison et al. 2013). The three additional articles considered descriptive survey data on stigma-related experiences (de Haan et al. 2002; Kapur et al. 2014; Del Vecchio et al. 2015).

To summarize the statistically significant findings from the association studies, an increase in perceived stigma among people at risk of psychosis between baseline and 1-year follow-up assessment was associated with more negative help-seeking attitudes for psychotherapy at follow-up ( $\mathrm{Xu}$ et al. 2016). Lower stigma stress among people at risk of psychosis was associated with more positive help-seeking attitudes towards both psychotherapy and psychiatric medication at baseline (Rüsch et al. 2013), whereas at 1-year follow up increased stigma stress since baseline was associated with more negative help-seeking attitudes towards psychotherapy only (Xu et al. 2016). People at risk of psychosis reported reduced internalized stigma following cognitive therapy, but so did those who did not receive therapy (Morrison et al. 2013). In quantitative descriptive studies, service-stigma was a reason for opposing psychiatric treatment among people with FEP, and shame was the main reason for nondisclosure of symptoms (de Haan et al. 2002). Concern that loved ones experiencing FEP would be labelled 'mad' was a frequent reason for relatives not contacting psychiatric services (Del Vecchio et al. 2015), and health professionals left young people who hear voices feeling 'not normal' (Kapur et al. 2014).

\section{Overall meta-synthesis}

The findings of the qualitative and quantitative results syntheses were combined in an overall meta-synthesis; Fig. 2 illustrates the resulting conceptual model. Overall there was close coherence between the qualitative and quantitative evidence, and quantitative findings fitted within the themes emerging from the qualitative synthesis. One contrasting finding was notable between qualitative and quantitative studies. Subsequently, based on quantitative findings only, the subtheme 'using personal resources to cope with stress of stigma' was added to the 'strategies' theme. This subtheme captured a strategy more reflective of resilience and support - considering a person's perceived social resources to overcome the harm of stigma - whereas the qualitative findings described strategies such as concealing or rejecting the stigmatized condition, or distancing oneself from the situation in which stigma and discrimination occurred.

\section{Additional analyses}

\section{Sensitivity analysis}

Eleven articles ( $n=4$ qualitative, $n=5$ quantitative, $n=2$ MMR) with MMAT ratings of $\leqslant 50 \%$ were excluded from the meta-synthesis. Subsequently, no MMR papers and only two quantitative papers remained. Subthemes added to the meta-synthesis model based on quantitative findings alone were lost, but all subthemes based on the qualitative results synthesis were retained as was the overall thematic structure of the meta-synthesis.

\section{Subgroup analyses}

Findings regarding stigma and pathways to care were examined among: (1) people at risk of developing psychotic disorders, $v$. those experiencing FEP; and (2) people affected by at-risk stages of psychotic disorders or FEP, $v$. significant other individuals. The third comparison planned between significant other individuals of people at risk of developing psychotic disorders $v$. significant other individuals of those experiencing FEP was prevented by the absence of studies considering the former perspective. The subgroup comparisons are summarized below; online Supplement 6 details the relative proportions of articles reporting on the themes and subthemes, by subgroup classification.

The first subgroup comparison considered 29 articles focusing on FEP populations and nine articles reporting on at-risk groups. The main themes 'characterizing difference negatively', 'negative reactions', and 'strategies' were reported in a similar manner across these groups. In contrast, findings reflecting 'sense of difference' were reported more often among FEP populations. Within this theme, however, the subtheme 'not being normal, something is wrong' was reported more frequently among at-risk groups, whereas the 'mental illness' subtheme was more commonly reported in FEP studies. The 'lack of knowledge and understanding' theme was reported in three studies only, but all considered FEP groups. Considering findings regarding service-specific stigma, deterring factors were reported among a higher proportion of FEP studies, whereas facilitating features were reported among a higher proportion of articles considering at-risk groups.

The second subgroup comparison considered twenty-four articles reporting on findings from the perspective of people experiencing FEP or at risk of psychosis ('affected persons') $v$. thirteen articles considering significant others' views. Findings reflecting 'negative reactions' and 'strategies' were reported in a similar manner across these groups. Findings reflecting 
'sense of difference' and 'characterizing difference negatively' were reported in a larger proportion of articles considering affected persons' perspectives. Stigma-related 'lack of knowledge and understanding' was only reported by three studies; none focused on affected persons' perspectives. Regarding servicespecific stigma, deterring influences were reported in comparable manner across the groups, but facilitating stigma-reducing factors were only reported by articles considering affected persons' perspectives.

\section{Discussion}

This is the first systematic review to examine stigma in relation to pathways to care for people in early stages of psychotic disorders (FEP populations and groups at risk of psychotic disorder), providing a comprehensive overview of current evidence in this area.

The primary review objective was to examine the relationship between stigma and pathways to care among people experiencing FEP or at risk of developing a psychotic disorder, and among significant other individuals supporting their pathways to care. Addressing this aim, our synthesis identified six themes in relation to stigma related to pathways to care among these groups: 'sense of difference', 'characterizing difference negatively', 'negative reactions (anticipated and experienced)', 'strategies', 'lack of knowledge and understanding', and 'service-related factors'. The conceptual model illustrating these themes alludes to the complex relationships between stigma and pathways to care, in terms of different processes through which the stigma-related influences can be observed (reflected in the synthesis themes and subthemes), how these relate to each other, and their connections with help-seeking and service use (see Fig. 2). Addressing the secondary objective of this review, these interconnections indicate potential mechanisms through which stigma can influence pathways to care. For example, a sense of difference often led people to anticipate negative labelling and judgemental reactions from others. Such outcomes were avoided through strategies like denial or non-disclosure, which could delay help-seeking. Another secondary objective was to examine how well stigma is researched in relation to pathways to care for FEP and at-risk populations. Assessing the methodological quality of the included studies indicated that qualitative studies in this field were generally of good quality, whereas the quality of quantitative and MMR studies was mixed. Quantitative studies were comprised by, for example, limited reporting on response rates and sample representativeness, and MMR studies by limited consideration of data integration. This indicates a paucity of high-quality quantitative and MMR evidence in this area.

Overall, the majority of evidence considered for our review was qualitative, with fewer quantitative studies. It was interesting to note some differences in these sets of data. Namely, qualitative evidence consistently indicated that stigma-related factors influenced processes associated with help-seeking and service contact both among FEP and at-risk groups. For example, stigma was reported as constantly present in help-seeking narratives (Ferrari et al. 2015), and impacting participants' beliefs and attitudes regardless of how knowledgeable families were about mental illness (Boydell et al. 2013). However, when examining quantitative associations between stigma and pathways to care, findings were often not statistically significant (Czuchta \& McCay, 2001; Compton \& Esterberg, 2005; Compton et al. 2009; Rüsch et al. 2013; Xu et al. 2016). This pattern might reflect the challenge of quantitatively capturing the influence of stigma, given how stigma is likely to be only one of many factors influencing help-seeking and healthcare use (Dockery et al. 2015) and its quantitatively assessed effect on help-seeking is small to moderate (Clement et al. 2015). Overall, it might be challenging to assess complex stigma-related influences and experiences through quantitative means (Compton et al. 2009), as the influence of stigma on processes along pathways to care is likely to vary between individuals and help-seeking contexts. Such complexity was alluded to among the quantitative studies in our review; it was suggested that stigma might contribute to longer DUPs through leading to greater perceived barriers to help-seeking, which might delay contact with mental health services (Compton \& Esterberg, 2005). Likewise, a more complete picture of stigma-related factors on help-seeking was obtained through considering not only perceived stigma, but also resources to cope with it (Rüsch et al. 2013; Xu et al. 2016). Qualitative approaches might be better suited to capture nuanced contextand person-dependent variablity in relation to stigma (Link et al. 2004; Clement et al. 2015), which may explain why the majority of evidence identified for this review was qualitative.

The, at times, contradictory insights obtained from qualitative and quantitative studies should not be considered reflective of one method producing more valid findings than the other. Rather, some points of divergence should be expected between findings obtained through these separate lines of inquiry, as they are underpinned by distinct epistemological paradigms [generally speaking, a positivist or post-positivist framework for quantitative research, and an interpretivist or constructivist tradition for qualitative research 
(Sale et al. 2002; Creswell, 2014)]. These approaches consequently provide insights reflecting different perspectives of a phenomena of interest (Whitley, 2007; Hasson-Ohayon et al. 2015). This variability can be considered a richness, and an enhanced understanding of a topic can be obtained through taking into account these differing perspectives (Creswell \& Plano Clarke, 2007; Creswell et al. 2011). Mixed-methods approaches could facilitate this type of deeper, multi-faceted, comprehensive understanding of the influences explored in this review, through explicitly contrasting and comparing overlaps and disparities in findings regarding stigma and pathways to care obtained through qualitative and quantitative means. Only two MMR studies were, however, identified for this review, indicating that such evidence is currently lacking.

Our review, focusing on FEP/at-risk states, corroborates the findings of past literature considering stigma and mental illness more generally. For example, stigma is suggested to constitute a barrier to preventing mental illness through public stigma, self-stigma, and shame contributing to reluctance to recognize early illness and avoidance of treatment (Corrigan, 2004; Rüsch \& Thornicroft, 2014), comparable to influences of stigma evident within our results synthesis. It has also been suggested that stigma can compromise prevention efforts through contributing to poor symptom recognition and knowledge about available treatments (Jorm et al. 2005; Rüsch \& Thornicroft, 2014), again, comparable to our findings. There are also similarities between our findings on strategies to avoid stigma-related reactions, and the findings of a recent systematic review on stigma and help-seeking among psychosis, non-psychosis and population samples, and a range of mental illnesses (Clement et al. 2015). Clement and colleagues also reported that concerns around not being normal were a common barrier to help-seeking among young people specifically, corresponding with our findings. These parallels indicate that stigma can operate in a comparable manner in relation to early stages of developing psychotic disorders, as explored in our review, as well as the more broadly conceptualized populations with mental illness and more chronic illness presentations examined in past literature.

Our subgroup comparisons provide further detailed insights regarding what is known about stigma for groups within this review's target population. For example, in terms of a 'sense of difference' that can instigate stigma processes, among at-risk groups this was commonly reported in terms of 'not being normal, something is wrong' whereas FEP groups more often reported this in relation to a more specific sense of 'mental illness'. Understanding these nuanced differences may help disentangle whether particular stigma-related factors are more relevant for certain subgroups.

These findings can inform efforts to mitigate stigma-related concerns that currently influence recognition of early difficulties and contribute to delayed helpseeking and access to care. For example, stigma-related fears linked to a general 'sense of difference' contributed to a reluctance to recognize signs of mental illness, disclose difficulties, and seek help. Consequently, mental health awareness efforts could focus on increasing the understanding of early signs of poor mental health. An improved awareness of how to interpret initial symptoms - what they might reflect and how they can be supported - could reduce help-seeking barriers relating to young people's fears of not being 'normal' or feeling 'weird' when symptoms emerge.

In addition to providing an evidence summary, we also identified gaps in the research on stigma and pathways to care among the review's target population. For example, the subgroup comparisons showed that facilitative destigmatizing features of services were reported more frequently among at-risk than FEP groups. Rather than indicating experiential differences, this could reflect an increased interest in exploring positive aspects of detecting and treating high-risk states of psychotic illnesses, given the debate of whether risks of stigmatization outweigh the benefits of at-risk labels/services (Corcoran et al. 2010; Woods et al. 2010; Yang et al. 2010), and a lack of research exploring this among FEP groups. Further, seemingly no studies have examined stigma and pathways to care among significant others of people in at-risk groups. Given the importance of caregivers and others in young people's help-seeking (Logan \& King, 2001), a more complete picture of stigma on early pathways to care could be achieved through understanding these influences among people supporting helpseeking efforts among people in at-risk groups.

\section{Limitations}

Our search strategy might not have captured all articles relevant for this review, and our choice of databases could have limited the search. However, we used a broad search strategy in multiple databases to avoid such issues. Relevant work might also have been excluded through our inclusion only of published, peer-reviewed papers; restrictions necessary to achieve a feasible volume of results to screen. The qualitative data synthesis was primarily conducted by the main author (P.C.G.), which could have biased the process. However, inclusion decisions and results synthesis were periodically discussed with another author (S.E-L.), and quotes from the included articles illustrating the themes are provided for transparency 
in online Supplement 5. Quantitative results could be synthesized using only a narrative approach; statistical pooling was prevented by the studies' methodological and conceptual heterogeneity. Also, the included studies were primarily conducted in high-income and Western countries, restricting the generalizability of the findings. Stigma-related influences can be stronger for people from certain cultural or ethnic backgrounds, due to culturally-linked beliefs and morals influencing interpretations of mental illness (Yang et al. 2007). Some studies in this review reported that people from given cultural or ethnic groups seemed particularly affected by stigma (e.g. Asian Pakistani families in UK setting; Connor et al. 2016). However, there were insufficient studies examining the role of culture or ethnic origin to enable subgroup comparisons. Additionally, the findings of this review should be interpreted in view of how other, non-stigma-related influences are also likely to constitute barriers on pathways to care (Dockery et al. 2015). These barriers can include, for example, structural/situational factors (e.g. issues with service location, timing or availability), financial reasons (e.g. cost of services, health insurance cover), low perceived service need, perceived ineffectiveness of services, or a preference to cope with a problem on their own (Kessler et al. 2001; Andrade et al. 2014).

\section{Conclusions}

The conceptual model that emerged from our results synthesis constitutes a comprehensive overview of the current qualitative and quantitative evidence-base regarding stigma and pathways to care among FEP and at-risk groups, illustrating the complex manner in which stigma-related processes can influence helpseeking and service contact at early stages of psychotic disorders. By contrasting our findings with previous literature, we identified similarities in the influence of stigma as observed in early stages of psychotic disorders $v$. established mental illnesses. These comparisons advance the understanding of the role of stigma on early pathways to care in emerging psychosis. The conceptual model derived from our findings can serve as a foundation for future research and efforts to mitigate the deterring influences on stigma on help-seeking and service contact.

\section{Supplementary material}

The supplementary material for this article can be found at https://doi.org/10.1017/S0033291717000344.

\section{Acknowledgements}

P.C.G. received funding support from the National Institute for Health Research (NIHR) Mental Health
Biomedical Research Centre at South London and Maudsley NHS Foundation Trust and King's College London. G.T. is supported by the NIHR under its Programme Grants for Applied Research scheme (Improving Mental Health Outcomes by Reducing Stigma and Discrimination: RP-PG-0606-1053). G.T. is supported by the National Institute for Health Research (NIHR) Collaboration for Leadership in Applied Health Research and Care South London at King's College London Foundation Trust. G.T. acknowledges financial support from the Department of Health via the National Institute for Health Research (NIHR) Biomedical Research Centre and Dementia Unit awarded to South London and Maudsley NHS Foundation Trust in partnership with King's College London and King's College Hospital NHS Foundation Trust. The views expressed in this paper are those of the author(s) and not necessarily those of the NHS, the NIHR, or the Department of Health. G.T. is supported by the European Union Seventh Framework Programme (FP7/2007-2013) Emerald project. S.E-L. is funded by the European Research Council under the European Union's Seventh Framework Programme (FP7/2007-2013)/ERC grant agreement no. 337673. K.R.L. was supported by funding from the Schizophrenia Research Institute, Australia, utilizing infrastructure funding from the New South Wales (NSW) Ministry of Health. The funders had no role in study design, data collection and analysis and interpretation, writing of the manuscript or decision to submit it for publication. We thank Gareth Hopkin for his contribution as a second rater during article screening and data extraction, Nicolas Rüsch for his feedback while developing the intended review procedures, and the authors of the included papers and other colleagues for their recommendations regarding additional publications to consider for this review.

\section{Declaration of Interest}

S.E-L. has received consulting fees from Lundbeck unrelated to this work.

\section{References}

Andrade LH, Alonso J, Mneimneh Z, Wells JE, Al-Hamzawi A, Borges G, Bromet E, Bruffaerts R, De Girolamo G, De Graaf R, Florescu S, Gureje O, Hinkov HR, Hu C, HUang Y, Hwang I, Jin R, Karam EG, Kovess-Masfety V, Levinson D, Matschinger H, O'Neill S, Posada-Villa J, Sagar R, Sampson NA, Sasu C, Stein D, Takeshima T, Viana MC, Xavier M, Kessler RC (2014). Barriers to mental health treatment: results from the WHO World Mental Health (WMH) surveys. Psychological Medicine 44, 1303-1317. 
Birchwood M, Macmillan F (1993). Early intervention in schizophrenia. Australian \& New Zealand Journal of Psychiatry 27, 374-378.

Birchwood M, McGorry P, Jackson H (1997). Early intervention in schizophrenia. British Journal of Psychiatry 170, $2-5$.

Boydell KM, Volpe T, Gladstone BM, Stasiulis E, Addington J (2013). Youth at ultra high risk for psychosis: using the Revised Network Episode Model to examine pathways to mental health care. Early Intervention in Psychiatry 7, 170-186.

Braun V, Clarke V (2006). Using thematic analysis in psychology. Qualitative Research in Psychology 3, 77-101.

Castle DJ, Singh SP (2015). Early intervention in psychosis: still the 'best buy'? British Journal of Psychiatry 207, 288292.

Centre for Reviews and Dissemination (2009). Systematic Reviews: CRD's Guidance for Undertaking Reviews in Health Care. Centre for Reviews and Dissemination, University of York: York, UK (http://www.york.ac.uk/inst/crd/pdf/ Systematic_Reviews.pdf). Accessed 4 March 2014.

Clement S, Schauman O, Graham T, Maggioni S, Evans-Lacko S, Bezborodovs N, Morgan C, Rüsch N, Brown JSL, Thornicroft G (2015). What is the impact of mental health-related stigma on help-seeking? A systematic review of quantitative and qualitative studies. Psychological Medicine 45, 11-27.

Compton MT, Esterberg ML (2005). Treatment delay in first-episode nonaffective psychosis: a pilot study with African American family members and the theory of planned behavior. Comprehensive Psychiatry 46, 291-295.

Compton MT, Goulding SM, Gordon TL, Weiss PS, Kaslow NJ (2009). Family-level predictors and correlates of the duration of untreated psychosis in African American first-episode patients. Schizophrenia Research 115, 338-345.

Connor C, Greenfield S, Lester H, Channa S, Palmer C, Barker C, Lavis A, Birchwood M (2016). Seeking help for first-episode psychosis: a family narrative. Early Intervention in Psychiatry 10, 334-345.

Corcoran CM, First MB, Cornblatt B (2010). The psychosis risk syndrome and its proposed inclusion in the DSM-V: a risk-benefit analysis. Schizophrenia Research 120, 16-22.

Corrigan PW (2004). How stigma interferes with mental health care. The American Psychologist 59, 614-625.

Creswell JW (2014). Research Design (International Student Edition): Qualitative, Quantitative, and Mixed Methods Approaches, 4th edn. SAGE Publications: Thousand Oaks, CA, US.

Creswell JW, Klassen AC, Plano Clark VL, Clegg Smith K (2011). Best Practices for Mixed Methods Research in the Health Sciences. Office of Behavioral and Social Sciences Research, National Institutes of Health (http://obssr.od.nih.gov/ mixed_methods_research/). Accessed 26 October 2012).

Creswell JW, Plano Clarke VL (2007). Designing and Conducting Mixed Methods Research. Sage Publications Ltd: London, UK.

Czuchta DM, McCay E (2001). Help-seeking for parents of individuals experiencing a first episode of schizophrenia. Archives of Psychiatric Nursing 15, 159-170. de Haan L, Peters B, Dingemans P, Wouters L, Linszen D (2002). Attitudes of patients toward the first psychotic episode and the start of treatment. Schizophrenia Bulletin 28, 431-442.

Del Vecchio V, Luciano M, Sampogna G, De Rosa C, Giacco D, Tarricone I, Catapano F, Fiorillo A (2015). The role of relatives in pathways to care of patients with a first episode of psychosis. The International Journal of Social Psychiatry 61, 631-637.

Dockery L, Jeffery D, Schauman O, Williams P, Farrelly S, Bonnington O, Gabbidon J, Lassman F, Szmukler G, Thornicroft G, Clement S (2015). Stigma- and non-stigma-related treatment barriers to mental healthcare reported by service users and caregivers. Psychiatry Research 228, 612-619.

EPPI-Centre (2010). EPPI-Centre Methods for Conducting Systematic Reviews. EPPI-Centre, Social Science Research Unit, Institute of Education, University of London: London, UK (https://eppi.ioe.ac.uk/cms/LinkClick.aspx? fileticket=hQBu8y4uVwI=\&tabid=88). Accessed 17 March 2014.

Ferrari M, Flora N, Anderson KK, Tuck A, Archie S, Kidd S, Mckenzie K (2015). The African, Caribbean and European (ACE) Pathways to Care study: a qualitative exploration of similarities and differences between African-origin , Caribbean-origin and European-origin groups in pathways to care for psychosis. BMJ Open 5.

Hasson-Ohayon I, Roe D, Yanos PT, Lysaker PH (2015). The trees and the forest: mixed methods in the assessment of recovery based interventions' processes and outcomes in mental health. Journal of Mental Health 25, 543-549.

Ho B, Andreasen NC (2001). Long delays in seeking treatment for schizophrenia. Lancet 357, 5-7.

Jorm AF, Blewitt KA, Griffiths KM, Kitchener BA, Parslow RA (2005). Mental health first aid responses of the public: results from an Australian national survey. BMC Psychiatry 5.

Kapur P, Hayes D, Waddingham R, Hillman S, Deighton J, Midgley N (2014). The experience of engaging with mental health services among young people who hear voices and their families: a mixed methods exploratory study. BMC Health Services Research 14.

Kessler RC, Berglund PA, Bruce ML, Koch JR, Laska EM, Leaf PJ, Manderscheid RW, Rosenheck RA, Walters EE, Wang PS (2001). The prevalence and correlates of untreated serious mental illness. Health Services Research 36, 987-1007.

Kohler C, Borgmann-Winter KE, Hurford I, Neustadter E, Yi J, Calkins ME (2014). Is prevention a realistic goal for schizophrenia? Current Psychiatry Reports 16, 439.

Lasalvia A, Zoppei S, Bonetto C, Tosato S, Zanatta G, Cristofalo D, De Santi K, Bertani M, Bissoli S, Lazzarotto L, Ceccato E, Riolo R, Marangon V, Cremonese C, Boggian I, Tansella M, Ruggeri M (2014). The role of experienced and anticipated discrimination in the lives of people with first-episode psychosis. Psychiatric Services 65, 1-7.

Link BG, Yang LH, Phelan JC, Collins PY (2004). Measuring mental illness stigma. Schizophrenia Bulletin 30, 511-541.

Logan D, King C (2001). Parental facilitation of adolescent mental health service utilization: a conceptual and 
empirical review. Clinical Psychology: Science and Practice 8, 319-333.

McGorry PD, Edwards J, Mihalopoulos C, Harrigan SM, Jackson HJ (1996). EPPIC: an evolving system of early detection and optimal management. Schizophrenia Bulletin 22, 305-326.

McGorry PD, Krstev H, Harrigan S (2000). Early detection and treatment delay: implications for outcome in early psychosis. Current Opinion in Psychiatry 13, 37-43.

Moher D, Liberati A, Tetzlaff J, Altman DG (2009). Preferred reporting items for systematic reviews and meta-analyses: the PRISMA statement. Annals of Internal Medicine 151, 264-269.

Morrison AP, Birchwood M, Pyle M, Flach C, Stewart SLK, Byrne R, Patterson P, Jones PB, Fowler D, Gumley AI, French P (2013). Impact of cognitive therapy on internalised stigma in people with at-risk mental states. British Journal of Psychiatry 203, 140-145.

Oliver S, Harden A, Rees R, Shepherd J, Brunton G, Garcia J, Oakley A (2005). An emerging framework for including different types of evidence in systematic reviews for public policy. Evaluation 11, 428-446.

Pescosolido BA, Martin JK (2015). The stigma complex. Annual Review of Sociology 41, 87-116.

Pluye P, Robert E, Cargo M, Bartlett G, O'Cathain A, Griffiths F, Boardman F, Gagnon MP, Rosseau MC (2011). Proposal: a mixed methods appraisal tool for systematic mixed studies reviews (http://mixedmethodsappraisaltool public.pbworks.com). Accessed 16 January 2014.

Popay J, Roberts H, Sowden A, Petticrew M, Arai L, Rogers M, Britten N, Roen K, Duffy S (2006). Guidance on the Conduct of Narrative Synthesis in Systematic Reviews. ESRC Methods Programme, University of Lancaster: Lancaster, UK. (http://www.lancaster.ac.uk/shm/research/nssr/ research/dissemination/publications.php).

Pope C, Mays N, Popay J (2007). Synthesising Qualitative and Quantitative Health Evidence: A Guide to Methods. McGrawHill International: Maidenhead, Berkshire, England.

Preti A, Cella M (2010). Randomized-controlled trials in people at ultra high risk of psychosis: a review of treatment effectiveness. Schizophrenia Research 123, 30-36.

Rose D, Willis R, Brohan E, Sartorius N, Villares C, Wahlbeck K, Thornicroft G (2011). Reported stigma and discrimination by people with a diagnosis of schizophrenia. Epidemiology and Psychiatric Sciences 20, 193-204.

Rüsch N, Heekeren K, Theodoridou A, Dvorsky D, Müller M, Paust T, Corrigan PW, Walitza S, Rössler W (2013).
Attitudes towards help-seeking and stigma among young people at risk for psychosis. Psychiatry Research 210, 1313-1315.

Rüsch N, Thornicroft G (2014). Does stigma impair prevention of mental disorders? British Journal of Psychiatry 204, 249-251.

Sale JEM, Lohfeld L, Brazil K (2002). Revisiting the quantitative-qualitative debate: implications for mixed-methods research. Quality and Quantity 36, 43-53.

Schomerus G, Angermeyer MC (2008). Stigma and its impact on help-seeking for mental disorders: what do we know? Epidemiologia e Psichiatria Sociale, 17, 31-37.

Stafford MR, Jackson H, Mayo-Wilson E, Morrison AP, Kendall T (2013). Early interventions to prevent psychosis: systematic review and meta-analysis. British Medical Journal 346, f185.

Tandon R, Nasrallah HA, Keshavan MS (2009). Schizophrenia, 'just the facts' 4 . Clinical features and conceptualization. Schizophrenia Research 110, 1-23.

Thornicroft G (2008). Stigma and discrimination limit access to mental health care. Epidemiologia e Psichiatria Sociale 17, 14-19.

Whitley R (2007). Mixed-methods studies. Journal of Mental Health 16, 697-701.

Woods SW, Walsh BC, Saksa JR, McGlashan TH (2010). The case for including Attenuated Psychotic Symptoms Syndrome in DSM-5 as a psychosis risk syndrome. Schizophrenia Research 123, 199-207.

Xu Z, Müller M, Heekeren K, Theodoridou A, Dvorsky D, Metzler S, Brabban A, Corrigan PW, Walitza S, Rössler W, Rüsch N (2016). Self-labelling and stigma as predictors of attitudes towards help-seeking among people at risk of psychosis: 1-year follow-up. European Archives of Psychiatry and Clinical Neuroscience 266, 79-82.

Yang LH, Anglin DM, Wonpat-Borja AJ, Opler MG, Greenspoon M, Corcoran C (2013). Public stigma associated with psychosis risk syndrome in a college population: implications for peer intervention. Psychiatric Services 64, 284-288.

Yang LH, Kleinman A, Link BG, Phelan JC, Lee S, Good B (2007). Culture and stigma: adding moral experience to stigma theory. Social Science and Medicine 64, 1524-1535.

Yang LH, Wonpat-Borja AJ, Opler MG, Corcoran C (2010). Potential stigma associated with inclusion of the psychosis risk syndrome in the DSM-V: an empirical question. Schizophrenia Research 120, 42-48. 